\title{
Periods of Limit Mixed Hodge Structures
}

\author{
Richard Hain
}

To Wilfred Schmid on the occasion of this 60th birthday

\section{Introduction}

The first goal of this paper is to explain some important results of Wilfred Schmid from his fundamental paper $[\mathbf{3 0}]$ in which he proves very general results which govern the behaviour of the periods of a of smooth projective variety $X_{t}$ as it degenerates to a singular variety. As has been known since classical times, the periods of a smooth projective variety sometimes contain significant information about the geometry of the variety, such as in the case of curves where the periods determine the curve. Likewise, information about the asymptotic behaviour of the periods of a variety as it degenerates sometimes contain significant information about the degeneration and the singular fiber. For example, the Hodge norm estimates, which are established in $[\mathbf{3 0}]$ and $[\mathbf{3}]$, describe the asymptotics of the Hodge norm of a cohomology class as the variety degenerates in terms of its monodromy. They are an essential ingredient in the study of the $L_{2}$ cohomology of smooth varieties with coefficients in a variation of Hodge structure $[37,4]$.

A second goal is to give some idea of how geometric and arithmetic information can be extracted from the limit periods, both in the geometric case and in the case of the limits of the mixed Hodge structures on fundamental groups of curves.

To get oriented, recall that if $X$ is a compact Riemann surface of genus $g$, then for each choice of a symplectic (w.r.t. the intersection form) basis $a_{1}, \ldots, a_{g}, b_{1}, \ldots, b_{g}$ of $H_{1}(X, \mathbb{Z})$, there is a basis $w_{1}, \ldots, w_{g}$ of the holomorphic differentials $H^{0}\left(X, \Omega^{1}\right)$ such that

$$
\int_{a_{j}} w_{k}=\delta_{j k}
$$

The $g \times g$ matrix

$$
\Omega:=\left(\int_{b_{j}} w_{k}\right)
$$

is called the period matrix of $X$. The classical Riemann bilinear relations assert that $\Omega$ is symmetric and that it has positive definite imaginary part. By the classical Torelli Theorem [17], the period matrix determines the Riemann surface up to isomorphism. 
A more conceptual way to view the period matrix is to note that the augmented period matrix $(I \mid \Omega)$ is the set of coordinates of the $g$-plane $H^{0}\left(X, \Omega^{1}\right)$ in the grassmannian of $g$ planes in the $2 g$-dimensional vector space $H^{1}(X, \mathbb{C})$ with respect to the integral basis $a_{1}, \ldots, b_{g}$ of $H_{1}(X, \mathbb{Z})$. The $g$-plane $H^{0}\left(C, \Omega^{1}\right)$ lies in the closed submanifold of the grassmannian consisting of $g$-planes isotropic with respect to the cup product.

\section{Quick Review of Hodge Theory}

2.1. The Hodge Theorem. For any complex manifold, we can define

$$
H^{p, q}(X):=\frac{\text { closed }(p, q) \text {-forms }}{(p, q) \text {-forms that are exact }} .
$$

This is simply the subspace of $H^{p+q}(X, \mathbb{C})$ consisting of classes that can be represented by a closed $(p, q)$-form.

For a general complex manifold, these do not give a decomposition of the cohomology of $X$. However, if $X$ is compact Kähler (for example, a smooth projective variety), then the natural mapping

$$
\bigoplus_{p+q=k} H^{p, q}(X) \rightarrow H^{k}(X, \mathbb{C})
$$

is an isomorphism. Denote the class in $H^{1,1}(X)$ of the Kähler form by $w$. The Hard Lefschetz Theorem states that if $\operatorname{dim} X=n$ and $k \geq 0$, then

$$
-\wedge w^{k}: H^{n-k}(X, \mathbb{C}) \rightarrow H^{n+k}(X, \mathbb{C})
$$

is an isomorphism. One can then define the primitive cohomology in degree $k$ by

$$
P H^{k}(X, \mathbb{C})=\operatorname{ker}\left\{\_\wedge w^{k+1}: H^{n-k}(X, \mathbb{C}) \rightarrow H^{n+k+2}(X, \mathbb{C})\right\} .
$$

The Hodge decomposition of $H^{k}(X)$ restrictions to give one of the primitive cohomology:

$$
P H^{k}(X, \mathbb{C}) \cong \bigoplus_{p+q=k} P H^{p, q}(X)
$$

where $P H^{p, q}(X)=H^{p, q}(X) \cap P H^{p+q}(X, \mathbb{C})$.

Define a form $Q: H^{n-k}(X) \otimes H^{n-k}(X) \rightarrow \mathbb{C}$ by

$$
Q(\xi, \eta)=\int_{X} \xi \wedge \eta \wedge w^{k}
$$

This form is always defined over $\mathbb{R}$ as $w$ is a real cohomology class, and over $\mathbb{Z}$ when $X$ is smooth projective and $w$ is the class of a hyperplane section.

The Riemann-Hodge bilinear relations generalize the classical Riemann bilinear relations for a compact Riemann surface. They state that

(i) $Q$ vanishes on $P H^{p, q}(X) \otimes P H^{r, s}(X)$ unless $p=s$ and $q=r$;

(ii) if $\xi \in P H^{p, q}(X)$ is non-zero, then

$$
i^{p-q}(-1)^{k(k-1) / 2} Q(\xi, \bar{\xi})>0,
$$

where $k=p+q$.

In degree 2, the Riemann-Hodge bilinear relations imply the Hodge Index Theorem.

The primitive cohomology $P^{k} H^{p, q}(X)$ together with the form $S:=(-1)^{k(k-1) / 2} Q$ is the prototypical example of a polarized Hodge structure of weight $k$.

Definition 1. A Hodge structure $V$ of weight $k$ consists of 
(i) a finitely generated abelian group $V_{\mathbb{Z}}$ and

(ii) a bigrading

$$
V_{\mathbb{C}}=\bigoplus_{p+q=k} V^{p, q}
$$

of its complexification, which satisfies $\overline{V^{p, q}}=V^{q, p}$.

A polarized Hodge structure of weight $k \in \mathbb{Z}$ is a Hodge structure $V$ of weight $k$ together with a $(-1)^{k}$ symmetric bilinear form

$$
S: V_{\mathbb{Z}} \otimes V_{\mathbb{Z}} \rightarrow \mathbb{Z}
$$

that satisfies:

(i) $S\left(V^{p, q}, V^{r, s}\right)=0$ unless $p=s$ and $q=r$,

(ii) if $v \in V^{p, q}$ is non-zero, then $i^{p-q} S(v, \bar{v})>0$.

The Hodge norm of $v \in V$ is defined by

$$
\|v\|^{2}=S(C v, \bar{v})
$$

where $C$ is the linear operator whose restriction to $V^{p, q}$ is $i^{p-q}$.

\section{Periods}

The Hodge filtration of a Hodge structure $V$ of weight $k$ is the decreasing filtration

$$
\cdots \supseteq F^{p} V \supseteq F^{p+1} V \supseteq F^{p+2} V \supseteq \cdots
$$

defined by

$$
F^{p} V:=\bigoplus_{s \geq p} V^{s, k-s}
$$

One can recover the bigrading from the Hodge filtration and the real structure by

$$
V^{p, k-p}=F^{p} V \cap \bar{F}^{k-p},
$$

where $\bar{F}^{\bullet}$ is the complex conjugate of the Hodge filtration.

The reason for working with the Hodge filtration rather than the bigrading is the observation of Griffiths [16] that the Hodge filtration varies holomorphically ${ }^{1}$ in families, whereas the $(p, q)$ pieces generally do not. Indeed, if $H^{p, q}$ varies holomorphically, then $H^{q, p}$ varies anti-holomorphically. So if both $H^{p, q}$ and $H^{q, p}$ vary holomorphically, then both are locally constant.

To be more precise, suppose that $f: X \rightarrow B$ is a family of compact Kähler manifolds. This means that $f$ is a proper holomorphic mapping, that $X$ is Kähler and that each fiber $X_{b}$ is smooth and has the induced Kähler structure. One has the local system (i.e., locally constant sheaf)

$$
\mathbb{H}^{k}:=R^{k} f_{*} \mathbb{Z}=\left\{H^{k}\left(X_{b}, \mathbb{Z}\right)\right\}_{b \in B}
$$

on $B$. We can complexify this to obtain a flat holomorphic vector bundle

$$
\mathcal{H}^{k}:=\mathbb{H}^{k} \otimes_{\mathbb{Z}} \mathcal{O}_{B}
$$

over $B$. Denote the natural flat connection on $\mathcal{H}^{k}$ by $\nabla$. Let

$$
\mathcal{F}^{p}:=\left\{F^{p} H^{k}\left(X_{b}\right)\right\}_{b \in B} \subseteq \mathcal{H}^{k} .
$$

\footnotetext{
${ }^{1}$ With respect to the locally constant structure coming from the lattice of integral cohomology.
} 
Fundamental results of Griffiths [16] assert that the $\mathcal{F}^{p}$ are holomorphic subbundles of $\mathcal{H}^{k}$ and that if one differentiates a section of $\mathcal{F}^{p}$ along a holomorphic vector field, then the result lies in $\mathcal{F}^{p-1}$ :

$$
\nabla: \mathcal{F}^{p} \rightarrow \mathcal{F}^{p-1} \otimes \Omega_{B}^{1}
$$

This is the prototypical example of a variation of Hodge structure. Replacing $\mathbb{H}^{k}$ by the bundle of primitive cohomology

$$
P \mathbb{H}^{k}:=\left\{P H^{k}\left(X_{b}\right)\right\}_{b \in B}
$$

yields the prototypical example of a polarized variation of Hodge structure - a variation of Hodge structure with an inner product $S$ which is parallel with respect to the connection and which polarizes each fiber.

DEFinition 2. A variation of Hodge structure of weight $k$ over a complex manifold $B$ is a $\mathbb{Z}$-local system $\mathbb{V}$ together with a flag

$$
\cdots \supseteq \mathcal{F}^{p} \supseteq \mathcal{F}^{p+1} \supseteq \cdots
$$

of holomorphic sub-bundles of the flat bundle $\mathcal{V}:=\mathbb{V} \otimes_{\mathbb{Z}} \mathcal{O}_{B}$ which satisfy:

(i) $\nabla: \mathcal{F}^{p} \rightarrow \mathcal{F}^{p-1} \otimes \Omega_{B}^{1}$,

(ii) Each fiber, endowed with the induced Hodge filtration, is a Hodge structure of weight $k$.

The variation is polarized if it has a $(-1)^{k}$-symmetric inner product which is parallel with respect to the flat structure and polarizes the Hodge structure on each fiber.

Given a polarized variation of Hodge structure $\mathbb{V}$ of weight $k$ over a base $B$, one has a period mapping

$$
\widetilde{B} \rightarrow\left\{\text { set of flags }\left\{F^{p}\right\} \text { that satisfy } S\left(F^{p}, F^{k-p+1}\right)=0\right\}
$$

defined on the universal covering of the base. The flags should be considered to live in some fixed fiber of $\mathbb{V} \rightarrow B$; other fibers being compared with this one using the flat structure. The flags should have the "same shape" as the Hodge filtration on this reference fiber.

Griffiths' results assert that this mapping is holomorphic and lies in the open subset $D$ of flags that satisfy

$$
i^{p-q} S(v, \bar{v})>0
$$

whenever $v$ is a non-zero element of $F^{p} \cap \bar{F}^{k-p}$ and $p+q=k$.

When the variation is $P \mathbb{H}^{k}$ this mapping really is a period mapping in the sense that the coordinates in the flag manifold of the image of $b \in \widetilde{B}$ will be given by periods over integral cycles of a basis of $P H^{k}\left(X_{b}\right)$ adapted to the Hodge filtration.

At this point it is useful to revisit the period mapping for curves.

ExAmPle 3. Fix a genus $g \geq 1$. To know the Hodge filtration on a compact Riemann surface of genus $g$, one simply has to know the location of the holomorphic differentials $F:=H^{1,0}\left(X_{b}\right)$ in $H^{1}(X, \mathbb{C})$ with respect to an integral symplectic basis. The polarization $S$ on $H^{1}\left(X_{b}\right)$ is the one given by cup product. The first Riemann bilinear relation says that $S(F, F)=0$.

Choose a base point $b_{o} \in B$. Let $H=H^{1}\left(X_{b_{o}}\right)$. The image of the period mapping is contained in the closed submanifold $Y$ of the grassmannian of $g$ planes in $H_{\mathbb{C}}$ consisting of those $g$-planes $F$ that satisfy $S(F, F)=0$. It is a complex 
manifold of dimension $g(g+1) / 2$. The period mapping $\widetilde{B} \rightarrow Y$ is holomorphic, and has image contained in the open subset

$$
U=\{F \in Y: i S(v, \bar{v})>0 \text { if } v \neq 0, v \in F\}
$$

of $Y$; it is a complex manifold. One can check that

$$
\begin{aligned}
U & =\text { the symmetric space of } \operatorname{Sp}_{g}(\mathbb{R}) \\
& =\operatorname{Sp}_{g}(\mathbb{R}) / U(g) \\
& =\text { Siegel's upper half plane } \mathfrak{h}_{g} \text { of rank } g \\
& =\{g \times g \text { symmetric complex matrices } \Omega \text { with } \operatorname{Im} \Omega>0\} .
\end{aligned}
$$

The period map takes $X_{b}$ to its period matrix $\Omega(b)$, described in the Introduction. That it is holomorphic is equivalent to the statement that $\Omega(b)$ depends holomorphically on $b \in B$.

The monodromy representation of the variation is a homomorphism

$$
\pi_{1}\left(B, b_{o}\right) \rightarrow \operatorname{Sp}_{g}(\mathbb{Z}) .
$$

The period mapping descends to the mapping

$$
B \rightarrow \operatorname{Sp}_{g}(\mathbb{Z}) \backslash \mathfrak{h}_{g}=\mathcal{A}_{g}
$$

to the moduli space of principally polarized abelian varieties that takes $b \in B$ to the jacobian of $X_{b}$.

\section{The Limit Hodge Filtration}

Schmid's work concerns the behaviour of the period mapping of a polarized variation of Hodge structure as the the Hodge structures degenerate. In the geometric case, this happens when the varieties $X_{b}$ degenerate to a singular variety. To keep things simple I'll only discuss the local case in one variable, which he considers in [30]. The several variable case is more subtle and is worked out in [30] and in $[3]$.

Suppose that $\mathbb{V} \rightarrow \Delta^{*}$ is a polarized variation of Hodge structure of weight $k$ over the punctured disk. The geometrically inclined may prefer to think of this as the local system of primitive cohomology of degree $k$ associated to a proper family $X \rightarrow \Delta$ over the disk, where $X$ is smooth and Kähler and where each fiber $X_{t}$ is smooth when $t \neq 0$.

Let

$$
T: V_{t_{o}} \rightarrow V_{t_{o}}
$$

be the monodromy operator associated to the positive generator of $\pi_{1}\left(\Delta^{*}, t_{o}\right)$. Landman in the geometric case, and Borel in general, (reproved by Schmid $[\mathbf{3 0}$, p. 245]) proved that the eigenvalues of $T$ are roots of unity, so that $T$ is quasi unipotent; that is, there exist integers $e$ and $m$ such that

$$
\left(T^{e}-I\right)^{m}=0 .
$$

By pulling $\mathbb{V}$ back along the $e$-fold covering $t \mapsto t^{e}$ of $\Delta^{*} \rightarrow \Delta^{*}$ we may assume that $T$ is unipotent. The logarithm of $T$ is then defined by the usual power series. Set

$$
N=\log T / 2 \pi i .
$$

This is nilpotent and preserves the polarization in the sense that

$$
S(N x, y)+S(x, N y)=0 .
$$


We shall view it as a flat section of the bundle End $\mathbb{V}$, so that it acts on all fibers, not just the one over $t_{o}$.

Denote the flat holomorphic vector bundle $\mathbb{V} \otimes_{\mathbb{Z}} \mathcal{O}_{\Delta^{*}}$ by $\mathcal{V}$ and its connection by $\nabla$.

Note that, unless $T$ has finite order, neither $T$ nor $N$ is a morphism of Hodge structures $V_{t_{o}} \rightarrow V_{t_{o}}$. One of Schmid's results, which is explained below, is that $N$ is compatible with the limit Hodge filtration and acts as an endomorphism of the "limit mixed Hodge structure", which allows one to relate Hodge theory and monodromy.

4.1. Deligne's Canonical Extension. In order to discuss the limit of the Hodge structure $V_{t}$ as $t \rightarrow 0$, we need to extend the flat bundle $\mathcal{V} \rightarrow \Delta^{*}$ to a holomorphic vector bundle $\widetilde{\mathcal{V}} \rightarrow \Delta$ over the entire disk. The way to do this is to use Deligne's canonical extension [6].

First note that any trivialization of $\mathcal{V}$ over $\Delta^{*}$ gives an extension of $\mathcal{V}$ to $\Delta$; the holomorphic sections of the extended bundle are those whose restriction to $\Delta^{*}$ are of the form

$$
\sum_{j} f_{j} \phi_{j}
$$

where each $f_{j}$ is holomorphic on $\Delta$, and $\phi_{1}, \ldots, \phi_{m}$ is a holomorphic frame that gives the trivialization over $\Delta^{*}$. Two framings of $\mathcal{V}$ over $\Delta^{*}$ give the same extension if and only if they differ by a holomorphic mapping $g: \Delta \rightarrow G L_{m}(\mathbb{C})$. It follows that the set of extensions of $\mathcal{V}$ to $\Delta$ can be identified with the homogeneous space

$$
G L_{m}\left(\mathcal{O}\left(\Delta^{*}\right)\right) / G L_{m}(\mathcal{O}(\Delta))
$$

which is large.

To construct Deligne's extension, we choose multivalued flat sections $\phi_{1}(t), \ldots, \phi_{m}(t)$ defined on $\Delta^{*}$. The extension is constructed by regularizing these. Specifically, set

$$
e_{j}(t)=\phi_{j}(t) t^{-N}
$$

where for a square matrix $A, t^{A}$ is defined to be $\exp (A \log t)$. The $e_{j}(t)$ comprise a single valued framing of $\mathcal{V}$ over $\Delta^{*}$ as each $\phi_{j}(t)$ changes to

$$
\phi_{j}(t) \cdot T
$$

when analytically continued around the circle, whereas $t^{-N}$ changes to

$$
\exp ((-\log t-2 \pi i) N)=e^{-2 \pi i N} t^{-N}=T^{-1} t^{-N}
$$

when continued around the origin. The framing $e_{j}$ determines Deligne's extension $\tilde{\mathcal{V}}$ of $\mathcal{V}$ to all of $\Delta$. With respect to Deligne's framing $e_{j}(t)$, the connection is

$$
\nabla=d-N \frac{d t}{t}
$$

In other words, the connection on $\widetilde{\mathcal{V}}$ is meromorphic with a simple pole at the origin and the residue of the connection at $t=0$ is $-N$. In particular, the extended connection has a regular singular point at the origin with nilpotent residue. This property characterizes Deligne's extension up to isomorphism. This fact, proved by Deligne in more generality in [6], is a consequence of the classical result about differential equations stated below, a proof of which can be found in [35, Chapt. II]. 
Lemma 4. Suppose that $A: \Delta \rightarrow$ End $V$ is holomorphic. If no two eigenvalues of $A_{0}:=A(0)$ differ by a non-zero integer (e.g., if $A_{0}$ is nilpotent), then there is a unique holomorphic function $P: \Delta \rightarrow$ Aut $V$ with $P(0)=\mathrm{id}_{V}$ such that each (local) solution $v: \Delta^{*} \rightarrow V$ of the differential equation

$$
t v^{\prime}(t)=v(t) A(t)
$$

is of the form

$$
v(t)=v_{0} t^{A_{0}} P(t) .
$$

Note that the monodromy operator $T$ is:

$$
v(t)=v_{0} t^{A_{0}} P(t) \mapsto v_{0} t^{A_{0}} e^{2 \pi i A_{0}} P(t)=v(t) \exp \left(2 \pi i P(t)^{-1} A_{0} P(t)\right) .
$$

The operator $\log T / 2 \pi i$ on the fiber over $t$ is thus right multiplication by

$$
N(t)=P(t)^{-1} A_{0} P(t) \in \text { Aut } V .
$$

The Deligne trivializing sections are therefore of the form

$$
v(t) t^{-N(t)}=v(t) P(t)^{-1} t^{-A_{0}} P(t)=v_{0} P(t),
$$

which converges to $v_{0}$ as $t \rightarrow 0$. This also implies that the function $P(t)$ is the difference between the Deligne framing and the given trivialization.

Corollary 5. If $A_{0}$ is nilpotent, then

$$
\lim _{t \rightarrow 0} v(t) t^{-A_{0}}=\lim _{t \rightarrow 0} v(t) t^{-N(t)}=v_{0}
$$

where the limit is taken along any angular ray.

Proof. The second equality was proved above. As for the first, we have

$$
v(t) t^{-A_{0}}=v_{0} t^{A_{0}} P(t) t^{-A_{0}} .
$$

If $A_{0}^{k+1}=0$, then there is a constant $C$ such that

$$
\left\|t^{A_{0}}\right\| \text { and }\left\|t^{-A_{0}}\right\| \leq C(\log 1 /|t|)^{k}
$$

when $0<|t| \leq R$, for some $R>0$. Writing $P(t)=I+\sum_{n \geq 1} P_{n} t^{n}$, we have

$$
\left\|t^{A_{0}} P(t) t^{-A_{0}}-I\right\| \leq 2 C|t|(\log 1 /|t|)^{k} \sum_{n=1}^{\infty}\left\|P_{n}\right\||t|^{n-1}
$$

which goes to zero along each angular ray.

This formula will prove useful in Section 6.2 when computing the period of the path $[0,1]$ in the limit MHS on the unipotent completion in the set of paths in $\mathbb{P}^{1}-\{0,1, \infty\}$ from 0 to 1 .

Remark 6 . Note that $v(t) t^{-A_{0}}$ is not, in general, single-valued on the punctured disk even though its limit as $t \rightarrow 0$ along each radial ray exists. 
4.2. The Nilpotent Orbit Theorem. Schmid's first main result is the Nilpotent Orbit Theorem. We shall state it as two separate results.

TheOREm 7 (Schmid). The Hodge sub-bundles $\mathcal{F}^{p}$ of $\mathcal{V}$ extend to holomorphic sub-bundles $\widetilde{\mathcal{F}}^{p}$ of Deligne's canonical extension $\widetilde{\mathcal{V}} \rightarrow \Delta$. That is, both $\widetilde{\mathcal{F}}^{p}$ and $\widetilde{\mathcal{V}} / \widetilde{\mathcal{F}}^{p}$ are vector bundles.

This shows that, when taken appropriately, the limit of the Hodge filtration exists. The limit Hodge filtration $\widetilde{\mathcal{F}}_{0}^{\bullet}$ is a filtration of the fiber $V_{0}$ of the canonical extension $\widetilde{\mathcal{V}}$ over 0 .

In the geometric case, the extended Hodge bundles can be constructed explicitly. This was done by Steenbrink in [31].

So far we have constructed a complex vector space $V_{0}$ and a Hodge filtration $F^{\bullet} V_{0}:=\widetilde{\mathcal{F}}_{0}^{p}$ on it. The polarization also extends to the central fiber as at $t^{N}$ preserves the inner product since $N$ preserves it infinitesimally by (2).

We can also construct an integral form $V_{\mathbb{Z}}$ of $V_{0}$. If the $\phi_{j}$ are integral, the integral lattice $V_{\mathbb{Z}}$ is simply the $\mathbb{Z}$-linear span of the $e_{j}(0)$. It is important to note, however, that except when the monodromy is trivial, $\left(V_{\mathbb{Z}}, F_{0}^{\bullet}\right)$ is not a Hodge structure even though it is a limit of Hodge structures.

In general, the integral structure depends on the holomorphic parameter $t$ chosen for the disk. This dependence is quite transparent, and is a straightforward consequence of Corollary 5.

Lemma 8. The lattice $V_{\mathbb{Z}}$ in $V_{0}$ depends only on the parameter $t$ to first order, and hence only on the tangent vector $\partial / \partial t$. The lattice corresponding to the tangent vector $\lambda \partial / \partial t$, where $\lambda$ is a sufficiently small non-zero complex number, is $\lambda^{N}$. $V_{\mathbb{Z}}$.

This extends to a "nilpotent orbit" over $\mathbb{C}^{*}$. Its fiber over $t \in \mathbb{C}^{*}$ consists of:

(i) the complex vector space $V_{0}$,

(ii) the limit Hodge filtration $F_{0}^{\bullet}$ on $V_{0}$,

(iii) the lattice $t^{N} V_{\mathbb{Z}}$ in $V_{0}$.

It has a flat connection given by the limit connection

$$
\nabla_{0}=d-N \frac{d t}{t}
$$

with respect to the constant trivialization given by $V_{0}$. It satisfies the Griffiths infinitesimal period relation (1).

The Deligne frame gives a connection preserving isomorphism

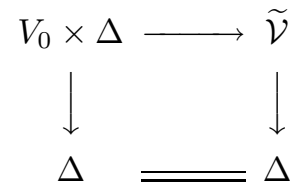

between $\widetilde{\mathcal{V}}$ and the restriction of the nilpotent orbit to a neighbourhood of the origin which is the identity on the fiber over the origin. The second part of Schmid's Nilpotent Orbit Theorem can be stated as follows.

TheOREM 9 (Schmid). The nilpotent orbit satisfies Griffiths infinitesimal period relation (1). There is an $\epsilon>0$ such that if $0<|t|<\epsilon$, then the fiber of the nilpotent orbit over $t$ is a Hodge structure with the same Hodge numbers 
as those of the original variation. Moreover, there is a holomorphic mapping $g: \Delta \rightarrow \operatorname{Aut}\left(V_{0}, S\right)$ such that $g(0)$ is the identity and $g$ carries the Hodge bundle of the nilpotent orbit onto the Hodge bundle of $\widetilde{\mathcal{V}}$.

So this gives us our first precise information about the asymptotics of periods of polarized variations of Hodge structure. Basically, the crudest model of a degeneration of Hodge structure is a nilpotent orbit. General degenerations are obtained from these by perturbing the Hodge filtration.

It is useful to think of the nilpotent orbit as being defined on the punctured tangent space $T_{0} \Delta-\{0\}$ of $\Delta$ at $t=0$. Then in a sufficiently small neighbourhood of the origin, the nilpotent orbit is a variation of Hodge structure that approximates the original variation. The nilpotent orbit can be thought of as the restriction of the canonical extension of the variation $\mathbb{V}$ to Spec $\mathbb{C}[t] /\left(t^{2}\right)$.

\section{The $\mathrm{SL}_{2}$-orbit Theorem}

Schmid's second main theorem, and the deeper of the two, is the $\mathrm{SL}_{2}$-orbit Theorem. It gives more precise information about how nilpotent orbits approximate degenerations of polarized variations of Hodge structure and is analogous to the Hard Lefschetz Theorem for the cohomology of a smooth projective variety. In fact, the $\mathrm{SL}_{2}$-orbit Theorem corresponds to the Hard Lefschetz Theorem for CalabiYau manifolds under mirror symmetry. ${ }^{2}$ It also leads naturally to mixed Hodge structures, which, like Hodge structures, have a lattice and Hodge filtration, but also have a weight filtration. In the case of a limit of Hodge structures, the weight filtration is constructed from the monodromy logarithm.

5.1. The Monodromy Weight Filtration. Suppose that $V$ is a finite dimensional vector space over a field of characteristic zero, and suppose that $N$ is a nilpotent endomorphism of $V$. Then there is a unique filtration $W(N)$ •

$$
\cdots \subseteq W_{n}(N) \subseteq W_{n+1}(N) \subseteq \cdots
$$

of $V$ such that

(i) $N\left(W_{n}(N)\right) \subseteq W_{n-2}(N)$ and

(ii) $N^{k}: W_{k} / W_{k-1} \rightarrow W_{-k} / W_{-k-1}$ is an isomorphism.

This is an easy exercise using the Jordan canonical form of $N$. It suffices to prove the result for a single Jordan block, where the result is evident.

In our case, the monodromy logarithm acts on $V_{0}$. We therefore have the monodromy weight filtration $W_{\bullet}(N)$ of $V_{0}$. In the present situation, we shift the filtration by the weight $k$ of the variation - define:

$$
W_{n} V_{0}=W_{k-n}(N) \text {. }
$$

\footnotetext{
${ }^{2}$ As Eduardo Cattani pointed out to me (cf. [2]), a precursor of this already appears in the papers $[\mathbf{3}, \mathbf{4}]$ of Cattani-Kaplan-Schmid. They prove a general result, a special case of which constructs out of $\left(H^{\bullet}(X), F^{\bullet}\right)$, the cohomology and Hodge filtration of a compact Kähler manifold $X$, a polarized variation of Hodge structure over the complexified Kähler cone of $X$. This can be restricted to the the $(p, p)$ part of $H^{\bullet}(X)$, and this is what should correspond to the polarized variation of Hodge structure of the degeneration of the mirror, as was pointed out to David Morrison by Cattani in 1994. Deligne [10] has also studied certain nilpotent orbits arising in mirror symmetry.
} 
Our central fiber now has the Hodge filtration $F^{\bullet} V_{0}$, the nilpotent orbit $t^{N} V_{\mathbb{Z}}$ of integral structures, and the monodromy weight filtration $W_{\bullet}$. One of the main consequences of the $\mathrm{SL}_{2}$-Orbit Theorem is:

TheOrem 10 (Schmid). For each $t \in \mathbb{C}^{*}$, the collection

$$
\left(t^{N} V_{\mathbb{Z}}, F_{0}^{\bullet}, W_{\bullet}\right)
$$

is a mixed Hodge structure. Moreover, $N: V_{0} \rightarrow V_{0}$ is defined over $\mathbb{Q}$ and is is a morphism of $\mathbb{Q}$-mixed Hodge structure of type $(-1,-1)$.

So, even if one is interested only in compact Kähler manifolds, one is inexorably lead to mixed Hodge structures when one studies their degeneration.

Definition 11 (Deligne). A mixed Hodge structure $V$ consists of a finitely generated abelian group $V_{\mathbb{Z}}$, an increasing filtration

$$
\cdots \subseteq W_{m-1} V_{\mathbb{Q}} \subseteq W_{m} V_{\mathbb{Q}} \subseteq W_{m+1} V_{\mathbb{Q}} \subseteq \cdots
$$

(called the weight filtration) of $V_{\mathbb{Q}}:=V_{\mathbb{Z}} \otimes_{\mathbb{Z}} \mathbb{Q}$, and a decreasing filtration

$$
\cdots \supseteq F^{p-1} V_{\mathbb{C}} \supseteq F^{p} V_{\mathbb{C}} \supseteq F^{p+1} V_{\mathbb{C}} \supseteq \cdots
$$

(called the Hodge filtration) of $V_{\mathbb{C}}:=V_{\mathbb{Z}} \otimes_{\mathbb{Z}} \mathbb{C}$. These are required to satisfy the condition that, for each $m$,

$$
\mathrm{Gr}_{m}^{W} V:=W_{m} V / W_{m-1} V
$$

with the induced Hodge filtration

$$
F^{p} \operatorname{Gr}_{m}^{W} V:=\operatorname{im}\left\{F^{p} V \cap W_{m} V \rightarrow \operatorname{Gr}_{m}^{W} V\right\}
$$

is a Hodge structure of weight $m$.

Deligne $[\mathbf{7}, \mathbf{8}]$ proved that the cohomology groups of every complex algebraic variety (not necessarily smooth or compact) have a mixed Hodge structure (MHS) that is natural with respect to morphisms.

This definition takes a while to digest, and it may be helpful to note that, up to some subtle issues of torsion in $\mathbb{V}_{\mathbb{Z}}$, mixed Hodge structures can be constructed as follows.

Let $\left(H^{m}\right)_{m \in \mathbb{Z}}$ be a collection of Hodge structures where $H^{m}$ has weight $m$ and all but a finite number of the $H^{m}$ are zero. Then their direct sum

$$
H=\bigoplus_{m} H^{m}
$$

is a mixed Hodge structure with the direct sum Hodge and weight filtrations:

$$
F^{p} H:=\bigoplus_{k} F^{p} H^{m}, \quad W_{m} H=\bigoplus_{k \leq m} H^{k} .
$$

Such a MHS is said to be split as it is the direct sum of Hodge structures. Very few of the MHSs encountered in life are split. Up to the torsion issues alluded to above, all MHSs $V$ with $\operatorname{Gr}_{m}^{W} V \cong H^{m}$ can be constructed as follows.

Let

$$
G=\left\{\phi \in \text { Aut } H_{\mathbb{C}} \text { that preserve } W_{\bullet} \text { and act trivially on all } \mathrm{Gr}_{m}^{W} H\right\} .
$$

This is a unipotent group. For each element of $g$ of $G$, we can define $V(g)$ to be the MHS with $V_{\mathbb{C}}=H_{\mathbb{C}}$, the same Hodge and weight filtrations (over $\mathbb{C}$ ), and lattice 
$g \cdot H_{\mathbb{Z}} \hookrightarrow H_{\mathbb{C}}$. The MHS obtained in this way, depends only on the double coset of $g^{-1}$ in

$$
G_{\mathbb{Z}} \backslash G / F^{0} G
$$

where

$$
G_{\mathbb{Z}}=G \cap \text { Aut } V_{\mathbb{Z}} \text { and } F^{0} G=\left\{\phi \in G: \phi \text { preserves } F^{\bullet}\right\} .
$$

In fact, this double coset space is the moduli space of MHSs $V$ together with an isomorphism $\mathrm{Gr}_{m}^{W} V \cong H^{m}$.

EXAMPLE 12. Let $A$ and $B$ be Hodge structures of weights $a$ and $b$, respectively, where $b<a$. The set of all mixed Hodge structures with weight graded quotients isomorphic to $A$ and $B$, together with these framings is

$$
\operatorname{Hom}\left(A_{\mathbb{C}}, B_{\mathbb{C}}\right) /\left(\operatorname{Hom}\left(A_{\mathbb{Z}}, B_{\mathbb{Z}}\right)+F^{0} \operatorname{Hom}(A, B)\right)
$$

as the group $G$ in this case is $\operatorname{Hom}\left(A_{\mathbb{C}}, B_{\mathbb{C}}\right)$. This gives the computation of $\operatorname{Ext}_{\text {Hodge }}^{1}(A, B)$ in the category of MHSs given by Carlson in $[\mathbf{1}]$.

5.2. The Hodge Norm Estimates. Another important consequence of the $\mathrm{SL}_{2}$-orbit Theorem is the Hodge norm estimates, which are important in applications to $L_{2}$-cohomology of algebraic varieties with coefficients in a polarized variation of Hodge structure - cf. [37] in the one-variable case, and [4] and [28] in general.

First note that the monodromy weight filtration is defined on the cohomology of each fiber and is preserved by the connection.

THEOREM 13 (Schmid). If $\mathbb{V}$ is a polarized variation of Hodge structure of weight $k$ over the punctured disk $\Delta^{*}$, then a flat section $v(t)$ lies in $W_{m}$ if and only if the square of its Hodge norm satisfies

$$
S(C v(t), \bar{v}(t))=O\left((\log (1 /|t|))^{m-k}\right)
$$

when $t$ goes to 0 along a radial ray. Here $C: V_{t} \rightarrow V_{t}$ is the operator that is multiplication by $i^{p-q}$ on $V_{t}^{p, q}$.

5.3. Topology and Geometry of the Limit MHS. Suppose that $f: X \rightarrow$ $\Delta$ is a proper holomorphic mapping from a Kähler manifold to the disk. Suppose that the fiber $X_{t}$ over $t \in \Delta$ is smooth whenever $t \neq 0$ and that $X_{0}$ is a reduced divisor with normal crossings. This implies that the monodromy operator $T \in$ Aut $H^{m}\left(X_{t}\right)$ is unipotent.

The local system

$$
\mathbb{V}:=R^{m}\left(\left.f\right|_{\Delta^{*}}\right)_{*} \mathbb{Z}
$$

underlies a polarized variation of Hodge structure of weight $m$ over $\Delta^{*}$. By Schmid's Theorems, for each choice of a non-zero tangent vector $\vec{v}$ of $\Delta$ at 0 , there is a limit mixed Hodge structure of $\mathbb{V}$, which one can think of as a MHS on the cohomology group $H^{m}\left(X_{\vec{v}}\right)$ of the first order deformation $X_{\vec{v}}$ of $X_{0}$ given by $\vec{v}$.

Basic results about the compatibility of this MHS with others associated to the degeneration follow from the geometric constructions of the limit MHS given by Clemens [5] and Steenbrink [31]. These compatibilities aid in extracting geometric and topological information from $H^{\bullet}\left(X_{\vec{v}}\right)$.

To begin to understand the limit MHS, $H^{m}\left(X_{\vec{v}}\right)$, it is helpful to think of $X_{v}$ as being built out of $X_{0}$. In order to explain this, we denote the normalization of 
$X_{0}$ by $\nu: \widetilde{X}_{0} \rightarrow X_{0}$. Denote the inverse image under $\nu: \widetilde{X}_{0} \rightarrow X_{0}$ of the singular locus of $X_{0}$ by $D$. It is a normal crossings divisor. Set

$$
X_{0}^{\prime}=\text { real oriented blowup of } \widetilde{X}_{0} \text { along } D
$$

which is a manifold with corners. One can think of each $X_{\vec{v}}$ as being obtained from $X_{0}^{\prime}$ by gluing, the data for which is given by the combinatorics of $D$, its local defining equation of $X_{0}$, and by $\vec{v} /|\vec{v}|$. There are therefore mappings

$$
\widetilde{X}_{0}-D \hookrightarrow X_{0}^{\prime} \rightarrow X_{\vec{v}} \rightarrow X_{0}
$$

which induce homomorphisms

$$
H^{m}\left(X_{0}\right) \stackrel{\beta}{\longrightarrow} H^{m}\left(X_{v}\right) \stackrel{\alpha}{\longrightarrow} H^{m}\left(\tilde{X}_{0}-D\right) .
$$

TheOrem 14. The mappings $\alpha$ and $\beta$ are both morphisms of MHS.

The statement that $\beta$ is a morphism is proved by both Clemens and Steenbrink. It is closely related to the Local Invariant Cycle Theorem, which is stated below. That $\alpha$ is a morphism follows from Steenbrink's construction of $H^{\bullet}\left(X_{\vec{v}}\right)$ in [31].

For each $t \in \Delta$, there is a restriction mapping

$$
j_{t}^{*}: H^{m}(X, \mathbb{Q}) \rightarrow H^{m}\left(X_{t}, \mathbb{Q}\right)
$$

which corresponds, via duality, to intersection with the fiber

$$
H_{n}\left(X,\left.X\right|_{\partial \Delta}, \mathbb{Q}\right) \rightarrow H_{n-2}\left(X_{t}, \mathbb{Q}\right)
$$

and to the mapping $\beta$ above after taking into account the fact that the inclusion $X_{0} \hookrightarrow X$ is a deformation retraction.

The following result was first established in the $\ell$-adic case by Deligne and then in the complex analytic case by Clemens [5] and Steenbrink [31]. Although not expressed in terms of limit Hodge theory, it is a non-trivial application of its existence and construction.

Theorem 15 (Local Invariant Cycle Theorem). If $t \neq 0$, then the image of $j_{t}^{*}$ is the space

$$
\operatorname{ker}\left\{(T-I): H^{m}\left(X_{t}, \mathbb{Q}\right) \rightarrow H^{m}\left(X_{t}, \mathbb{Q}\right)\right\}
$$

of invariant cohomology classes.

5.4. Degenerations of Curves. In this section, we give a relatively simple example to illustrate how geometric information can be extracted from the limit mixed Hodge structure. To get the full power from this theory, one needs to combine Schmid's results with the complementary results of Clemens [5] and the explicit constructions of Steenbrink [31] surveyed in the previous paragraph.

Suppose that $C \rightarrow \Delta$ is a stable degeneration of compact Riemann surfaces of genus $g$. The total space $C$ is assumed to be smooth and the fiber $C_{t}$ over $t$ is assumed to be smooth when $t \neq 0$. The central fiber is assumed to be reduced and stable (i.e., its automorphism group is finite.)

Let $B$ be the set of the homology classes of the vanishing cycles. Then, using the Picard-Lefschetz formula, one sees that the monodromy is unipotent and satisfies $(T-I)^{2}=0$. This implies that $N=T-I$ and that the monodromy weight filtration has length 3 :

$$
0 \subseteq W_{0} H^{1}\left(C_{t}\right) \subseteq W_{1} H^{1}\left(C_{t}\right) \subseteq W_{2} H^{1}\left(C_{t}\right)=H^{1}\left(C_{t}\right) .
$$


The defining properties of the monodromy weight filtration imply that

$$
W_{0} H^{1}\left(C_{t}\right)=\operatorname{im} N \text { and } W_{1} H^{1}\left(C_{t}\right)=\operatorname{ker} N .
$$

The Picard-Lefschetz formula implies that $W_{0} H^{1}\left(C_{t}\right)$ is spanned by the Poincaré duals of the vanishing cycles.

Fix a tangent vector $\vec{v}$ of 0 in $\Delta$ and denote the corresponding limit MHS by $H^{1}\left(C_{\vec{v}}\right)$.

Denote the normalization of $C_{0}$ by $\widetilde{C}_{0}$. Let $D$ be the inverse image in $\widetilde{C}_{0}$ of the double points of $C_{0}$. Results of the previous paragraph imply that the natural morphisms

$$
H^{1}\left(C_{0}\right) \stackrel{\beta}{\longrightarrow} H^{1}\left(C_{\vec{v}}\right) \stackrel{\alpha}{\longrightarrow} H^{1}\left(C_{0}-D\right)
$$

are morphisms of MHS.

The fact that $F^{0} H^{1}\left(C_{\vec{v}}\right)=H^{1}\left(C_{\vec{v}}, \mathbb{C}\right)$ and $F^{2} H^{1}\left(C_{\vec{v}}\right)=0$ imply that

$$
\mathrm{Gr}_{m}^{W} H^{1}\left(C_{\vec{v}}\right)
$$

is of type $(0,0)$ when $m=0$, of type $(1,1)$ when $m=2$, and is a polarized Hodge structure of with Hodge numbers $(1,0)$ and $(0,1)$ when $m=1$. Basic topology implies that $\beta$ injective, from which it follows that $\beta$ induces a natural isomorphism

$$
\operatorname{Gr}_{1}^{W} H^{1}\left(C_{\vec{v}}\right) \cong H^{1}\left(\widetilde{C}_{0}\right) \cong \bigoplus_{\substack{\text { components } \\ E \text { of } C_{0}}} H^{1}(E)
$$

of polarized Hodge structures. It also implies that $W_{0} H^{1}\left(C_{\vec{v}}\right)$ is subspace of $H^{1}\left(C_{\vec{v}}\right)$ generated by the Poincaré duals of the vanishing cycles and that $\beta$ induces an isomorphism

$$
W_{1} H^{1}\left(C_{\vec{v}}\right) \cong H^{1}\left(C_{0}\right)
$$

The classical Torelli Theorem, the fact that the theta divisor of the jacobian of a smooth curve is irreducible and the semi-simplicity of polarized Hodge structures of weight 1 imply that the polarized limit MHS, $H^{1}\left(C_{\vec{v}}\right)$, determines the normalization of $C_{0}$. Using this, and by approximating the period mapping by the nilpotent orbit, one can give proofs of weak versions (i.e., $\bmod t$ ) of the two results $[\mathbf{1 3}$, Cor. 3.2 , Cor 3.8] in Fay's book.

If the normalization of $C_{0}$ is connected, then one can determine the divisor classes in Jac $\widetilde{C}_{0}$ of the pairs of points that are identified to obtain $C_{0}$. This information is extracted from the extension

$$
0 \rightarrow W_{0} H^{1}\left(C_{\vec{v}}\right) \rightarrow W_{1} H^{1}\left(C_{\vec{v}}\right) \rightarrow \operatorname{Gr}_{1}^{W} H^{1}\left(C_{\vec{v}}\right) \rightarrow 0 .
$$

using the work of Carlson [1] and the isomorphism (3). This implies that when $C_{0}$ is irreducible, the limit MHS $H^{1}\left(C_{\vec{v}}\right)$ determines $C_{0}$ up to isomorphism.

On the other hand, if the dual graph of $C_{0}$ is a tree, then all vanishing cycles are trivial in homology, and the Picard-Lefschetz formula implies that $N=0$. In this case, the monodromy weight filtration is trivial, and the limit $\operatorname{MHS} H^{1}\left(C_{\vec{v}}\right)$ is a polarized Hodge structure of weight 1. By the argument above, this determines the non-rational components of $\widetilde{C}_{0}$ up to isomorphism. But since there is no extension data, it gives no information about how to reassemble $C_{0}$ from its irreducible components. In this case, one can use the limit MHS on the truncation of the group 
ring of the fundamental group of the smooth fiber by the 4th power of its augmentation ideal ${ }^{3}$ to determine $C_{0}$ up to finite ambiguity using the limit mixed Hodge structure on the fundamental group which is constructed in [20]. A proof of this assertion is not published, but most of the technical constructions needed to prove it are worked out in detail in the doctoral thesis of Rainer Kaenders [24]. More generally, I believe that for all stable degenerations of curves, the limit mixed Hodge structure on the truncation of the integral group ring of the fundamental group of the smooth fiber by the 5 th power of its augmentation ideal should determine $C_{0}$ up to finite ambiguity.

\section{Periods of $\pi_{1}\left(\mathbb{P}^{1}-\{0,1, \infty\}, \overrightarrow{01}\right)$}

In this section, we sketch a fundamental example of computing the periods of a limit MHS on the fundamental group of a the thrice punctured line. The periods turn out to be Euler's the mixed zeta numbers (cf. [36], [15]).

6.1. Hodge Theory of Homotopy Groups. This is a very brief introduction to the Hodge theory of homotopy groups of complex algebraic varieties.

First, suppose that $\pi$ is a discrete group. For a commutative ring $R$, denote the group algebra of $\pi$ over $R$ by $R \pi$. There is a natural augmentation

$$
\epsilon: R \pi \rightarrow R
$$

defined by taking $\sum r_{j} g_{j}$ to $\sum r_{j}$, where each $r_{j} \in R$ and $g_{j} \in \pi$. The augmentation ideal $J_{R}$ is the kernel of the augmentation. The mapping

$$
J_{R} / J_{R}^{2} \rightarrow H_{1}(\pi, R) \quad(g-1)+J_{R}^{2} \rightarrow[g]
$$

is a group isomorphism, which can be thought of as a kind of Hurewicz isomorphism.

Theorem 16 (Morgan [29], Hain [19]). If $X$ is a complex algebraic variety and $x \in X$, then for each $s \geq 0$, there is a natural MHS on the truncated group ring $\mathbb{Z} \pi_{1}(X, x) / J^{s+1}$. These form an inverse system in the category of $M H S$. The mixed Hodge structure on $\mathrm{J} / \mathrm{J}^{2}$ is dual to Deligne's $M H S$ on $H^{1}(X)$.

These mixed Hodge structures are constructed using Chen's iterated integrals.

Suppose that $M$ is a manifold and that $w_{1}, \ldots, w_{r}$ are smooth $\mathbb{C}$-valued 1 -forms on $M$. For each piecewise smooth path $\gamma:[0,1] \rightarrow M$, we can define

$$
\int_{\gamma} w_{1} \ldots w_{r}=\int \cdots \int_{0 \leq t_{1} \leq \cdots \leq t_{r} \leq 1} f_{1}\left(t_{1}\right) \cdots f_{r}\left(t_{r}\right) d t_{1} \ldots d t_{r},
$$

where $\gamma^{*} w_{j}=f_{j}(t) d t$ for each $j$. This is viewed as a $\mathbb{C}$-valued function

$$
\int_{\gamma} w_{1} \ldots w_{r}: P M \rightarrow \mathbb{C}
$$

on the the space of piecewise smooth paths in $M$. When $r=1, \int_{\gamma} w$ is just the usual line integral. An iterated integral is any function $P M \rightarrow \mathbb{C}$ which is a linear combination of a constant function and basic iterated integrals

$$
\int_{\gamma} w_{1} \ldots w_{r}
$$

An exposition of the construction of the MHS in $J / J^{s+1}$ can be found in [18].

\footnotetext{
${ }^{3}$ The mixed Hodge structure on the fundamental groups is discussed in the next section. Also, to apply it, one needs to choose a section $\sigma: \Delta \rightarrow C$ of base points.
} 
6.2. The MHS on $\pi_{1}\left(\mathbb{P}^{1}-\{0,1, \infty\}, t\right)$. One can describe the MHS on the $J$-adic completion

$$
\mathbb{Q} \pi_{1}\left(\mathbb{P}^{1}-\{0,1, \infty\}, t\right)^{\wedge}:=\lim _{\leftarrow} \mathbb{Q} \pi_{1}\left(\mathbb{P}^{1}-\{0,1, \infty\}, t\right) / J^{s} .
$$

of $\pi_{1}\left(\mathbb{P}^{1}-\{0,1, \infty\}, t\right)$ quite directly.

Consider the non-commutative power series ring

$$
A:=\mathbb{C}\left\langle\left\langle X_{0}, X_{1}\right\rangle\right\rangle
$$

freely generated by the indeterminates $X_{0}$ and $X_{1}$. Set

$$
w_{0}=\frac{d z}{z} \text { and } w_{1}=\frac{d z}{1-z} .
$$

Now consider the $A$-valued iterated integral

$$
T=1+\int w_{0} X_{0}+\int w_{1} X_{1}+\cdots+\int w_{j_{1}} \ldots w_{j_{r}} X_{j_{1}} \ldots X_{j_{r}}+\cdots
$$

It is not difficult to use the definition of iterated integrals to show that the value $T(\gamma)$ of this on a path $\gamma$ depends only on its homotopy class relative to its endpoints. In addition, one can show that if $\alpha$ and $\beta$ are composable paths, then ${ }^{4}$

$$
T(\alpha \beta)=T(\alpha) T(\beta) .
$$

For each $t \in \mathbb{P}^{1}-\{0,1, \infty\}$, we can thus define a homomorphism

$$
\pi_{1}\left(\mathbb{P}^{1}-\{0,1, \infty\}, t\right) \rightarrow \text { the group of units of } A
$$

by taking the class of the loop $\gamma$ based at $t$ to $T(\gamma)$. This induces a homomorphism

$$
\Theta_{t}: \mathbb{C} \pi_{1}\left(\mathbb{P}^{1}-\{0,1, \infty\}, t\right)^{\wedge} \rightarrow A
$$

which can easily be shown to be an isomorphism by using universal mapping properties of free groups and free algebras.

We can use this to construct a MHS on $\mathbb{Q} \pi_{1}\left(\mathbb{P}^{1}-\{0,1, \infty\}, t\right)^{\uparrow}$. Give each generator $X_{j}$ type $(-1,-1)$. Extend this bigrading to all monomials in $A$ in the standard way - the monomial $X_{I}$ will have Hodge type $(-|I|,-|I|)$ where $I=$ $\left(i_{1}, \ldots, i_{r}\right)$ is a multi index and $|I|$ is its length $r$. Thus $X_{I}$ has weight $-2|I|$. It is natural to define

$$
W_{m} A=\text { the closure of the span of } X_{I} \text { where }-2|I| \leq m
$$

(which is the $\ell$ th power of the maximal ideal when $m=-2 \ell$ ) and

$$
F^{p} A=\text { the span of the } X_{I} \text { where }-|I| \geq p
$$

which is finite dimensional for all $p$. Pulling back these filtrations along $\Theta_{t}$ defines the standard MHS on $\mathbb{Q} \pi_{1}\left(\mathbb{P}^{1}-\{0,1, \infty\}, t\right)^{\wedge}$.

The periods of the MHS on $\mathbb{Q} \pi_{1}\left(\mathbb{P}^{1}-\{0,1, \infty\}, t\right)^{\wedge}$ are the coefficients of the monomials $X_{I}$ in the power series $T(\gamma)$, where $\gamma \in \pi_{1}\left(\mathbb{P}^{1}-\{0,1, \infty\}, t\right)$.

\footnotetext{
${ }^{4}$ Note that I use the topologists convention that if $\alpha, \beta:[0,1] \rightarrow X$ are two paths with $\alpha(1)=\beta(0)$, then $\alpha \beta$ is the path obtained by first traversing $\alpha$, then $\beta$. This is the opposite of the convention used by many papers in this field.
} 
REMARK 17. In the Hodge theory of complex varieties, the periods are typically (local) coordinates in an appropriate moduli space of MHS, such as the one

$$
G_{\mathbb{Z}} \backslash G / F^{0} G
$$

discussed near the end of Section 5.1. However, in more arithmetic situations, where the MHS arises from a variety defined (say) over $\mathbb{Q}$, the de Rham invariant together with its Hodge and weight filtrations has a natural $\mathbb{Q}$-form. In this case, one usually takes the periods to be the integrals over rational (betti) cycles of a basis of the $\mathbb{Q}$-de Rham version of the invariant which is adapted to the Hodge and weight filtrations.

This is the case above as $\left(\mathbb{P}^{1}-\{0,1, \infty\}, t\right)$ is defined over $\mathbb{Q}$ when $t$ is $\mathbb{Q}$ rational. There is an algebraic de Rham theorem for the unipotent fundamental group in this case (cf. [21]), which is particularly easy to describe in the case above. The dual of $\mathbb{C} \pi_{1}\left(\mathbb{P}^{1}-\{0,1, \infty\}, t\right) / J^{N+1}$ is, by Chen's $\pi_{1}$ de Rham Theorem (or elementary arguments in this case),

$$
V_{\mathbb{C}}^{\mathrm{DR}}:=\left\{\sum_{r \leq N} a_{I} \int w_{i_{1}} \ldots w_{i_{r}}: I \subset\{0,1\}^{r},|I|=r, a_{I} \in \mathbb{C}\right\} .
$$

This has the natural $\mathbb{Q}$-form

$$
V_{\mathbb{Q}}^{\mathrm{DR}}:=\left\{\sum_{r \leq N} a_{I} \int w_{i_{1}} \ldots w_{i_{r}}: I \subset\{0,1\}^{r},|I|=r, a_{I} \in \mathbb{Q}\right\} .
$$

The basis of $\mathbb{C} \pi_{1}\left(\mathbb{P}^{1}-\{0,1, \infty\}, t\right) / J^{N+1}$ dual to basis $\left\{\int w_{i_{1}} \ldots w_{i_{r}}\right\}$ of $V_{\mathbb{Q}}^{\mathrm{DR}}$ is the set of monomials $X_{I}:=X_{i_{1}} \ldots X_{i_{r}}$. The periods of the MHS on $\mathbb{C} \pi_{1}\left(\mathbb{P}^{1}-\right.$ $\{0,1, \infty\}, t) / J^{N+1}$ are thus the integrals of the $\int w_{i_{1}} \ldots w_{i_{r}}$ over elements of $\mathbb{Q} \pi_{1}\left(\mathbb{P}^{1}\right.$ $\{0,1, \infty\}, t) / J^{N+1}$.

6.3. The MHS on $\pi_{1}\left(\mathbb{P}^{1}-\{0,1, \infty\}, \overrightarrow{01}\right)$. In general, the periods of the MHS on $\pi_{1}\left(\mathbb{P}^{1}-\{0,1, \infty\}, t\right)$ are difficult to compute as the values of the coefficients of $T$ on paths based at $t$ do not appear to be readily recognizable numbers when $t$ is general. However, if we let $t$ approach 0, the periods (or their asymptotics) become more recognizable as we shall explain.

When $t$ goes to 0 , the MHS on $\mathbb{Q} \pi_{1}\left(\mathbb{P}^{1}-\{0,1, \infty\}, t\right)^{-}$degenerates as all of the integrals of length $\geq 2$ that begin or end with $w_{0}$, such as $\int w_{0} w_{1}$, will diverge. (The MHS on the fundamental group can degenerate when the variety becomes singular, and also when the base point runs off the edge of the space as it is here.) Just as there is Schmid's theory of limits of Hodge structures, there is a theory of limits of MHSs, at least in the geometric case [32]. (There is a fledgling theory in the abstract case too, being developed by Kaplan and Pearlstein [25].) We will compute the periods of the limit mixed Hodge structure as $t \rightarrow 0$ with respect to the tangent vector $0 \overrightarrow{1}:=\partial / \partial t$, where $t$ is the standard parameter on $\mathbb{P}^{1}$.

Deligne [9] introduced the idea of the fundamental group of (say) an affine curve $C$ with base point a non-zero tangent vector $\vec{v}$ at one of the cusps $P$. It is simple and elegant. The fundamental group $\pi_{1}(C, \vec{v})$ is the set of homotopy classes of loops $\gamma$ in $C \cup\{P\}$ based at $P$, that leave $P$ with velocity vector $\vec{v}$ and return to $P$ with velocity vector $-\vec{v}$. It is also required that $\gamma$ does not return to $P$ when 
$0<t<1$. It is naturally isomorphic to the standard fundamental group. ${ }^{5}$ This will carry a limit MHS associated to the tangent vector $\vec{v}$.

Definition 18. For integers $n_{1}, \ldots, n_{r}$, where $n_{r}>1$, define

$$
\zeta\left(n_{1}, \ldots, n_{r}\right)=\sum_{0<k_{1}<\cdots<k_{r}} \frac{1}{k_{1}^{n_{1}} k_{2}^{n_{2}} \ldots k_{r}^{n_{r}}} .
$$

These numbers generalize the classical values of the Riemann zeta function at integers $>1$ and were first considered by Euler. They have recently resurfaced in the works of Zagier [36] and Goncharov [15]. Their $\mathbb{Q}$-linear span in $\mathbb{R}$ is a subalgebra MZN due to combinatorial identities such as

$$
\zeta(n) \zeta(m)=\zeta(n, m)+\zeta(m, n)+\zeta(m+n) .
$$

Since $\zeta(2)=\pi^{2} / 6$,

$$
\mathrm{MZN}_{\mathbb{C}}:=\mathrm{MZN} \oplus i \pi \mathrm{MZN}
$$

is a $\mathbb{Q}$-subalgebra of $\mathbb{C}$.

Mixed zeta numbers can be expressed as iterated integrals (cf. [36]):

$$
\zeta\left(n_{1}, \ldots, n_{r}\right)=\int_{0}^{1} w_{1} \overbrace{w_{0} \ldots w_{0}}^{n_{1}-1} w_{1} \overbrace{w_{0} \ldots w_{0}}^{n_{2}-1} w_{1} \ldots w_{1} \overbrace{w_{0} \ldots w_{0}}^{n_{r}-1} .
$$

Here the path of integration is along the unit interval, and the integral converges if and only if $n_{r}>1$. This identity follows directly from the definition of iterated integrals using the following slightly more general form:

$$
\int_{0}^{x} w_{1} \overbrace{w_{0} \ldots w_{0}}^{n_{1}-1} w_{1} \overbrace{w_{0} \ldots w_{0}}^{n_{2}-1} w_{1} \ldots w_{1} \overbrace{w_{0} \ldots w_{0}}^{n_{r}-1}=\sum_{0<k_{1}<\ldots<k_{r}} \frac{x_{1}^{k_{r}}}{k_{1}^{n_{1}} k_{2}^{n_{2}} \ldots k_{r}^{n_{r}}} .
$$

The basic result we wish to explain is the following "folk theorem." Goncharov in $[\mathbf{1 5}]$ has recently written down a proof of a considerably more refined statement.

THEOREM 19. The space of periods of the limit mixed Hodge structure on $\mathbb{Q} \pi_{1}\left(\mathbb{P}^{1}-\{0,1, \infty\}, \overrightarrow{01}\right)^{\wedge}$ is precisely $\mathrm{MZN}_{\mathbb{C}}$.

It is convenient to give a proof of this theorem by appealing to the MHS on spaces of paths. Denote the space of piecewise smooth paths from $a$ to $b$ in $\mathbb{P}^{1}-$ $\{0,1, \infty\}$ by $P_{a, b}\left(\mathbb{P}^{1}-\{0,1, \infty\}\right)$. Endow it with the compact open topology. Then

$$
H_{0}\left(P_{a, b}\left(\mathbb{P}^{1}-\{0,1, \infty\}\right), \mathbb{Q}\right)
$$

is the $\mathbb{Q}$-vector space generated by the homotopy classes of paths in $\mathbb{P}^{1}-\{0,1, \infty\}$ that go from $a$ to $b$. It is a module over $\mathbb{Q} \pi_{1}\left(\mathbb{P}^{1}-\{0,1, \infty\}, a\right)$ and can be completed in the $J$-adic topology. Denote this completion by

$$
H_{0}\left(P_{a, b}\left(\mathbb{P}^{1}-\{0,1, \infty\}\right), \mathbb{Q}\right)^{\Upsilon} \text {. }
$$

(It is also a $\mathbb{Q} \pi_{1}\left(\mathbb{P}^{1}-\{0,1, \infty\}, b\right)$ module. The completion with respect to this action is identical with the completion above.) As in the case where $a=b$, we can define a MHS on it by pulling back the Hodge and weight filtrations of $A$ along the mapping

$$
\Theta_{a, b}: H_{0}\left(P_{a, b}\left(\mathbb{P}^{1}-\{0,1, \infty\}\right), \mathbb{Q}\right)^{\wedge} \rightarrow A
$$

\footnotetext{
${ }^{5}$ An elegant way to prove this is to note that $\pi_{1}(C, \vec{v})$ is naturally isomorphic to $\pi_{1}(\widetilde{C},[\vec{v}])$ where $\widetilde{C}$ is the real oriented blowup of $C$ at $P$ and $[\vec{v}]$ is the point on the exceptional circle $\partial \widetilde{C}$ corresponding to the oriented ray determined by $\vec{v}$.
} 
induced by taking $\gamma$ to $T(\gamma)$, which is an isomorphism. The natural mappings

$$
\begin{aligned}
H_{0}\left(P_{a, b}\left(\mathbb{P}^{1}-\{0,1, \infty\}\right), \mathbb{Q}\right)^{\wedge} \otimes H_{0}\left(P_{b, c}\left(\mathbb{P}^{1}-\{0,1, \infty\}\right), \mathbb{Q}\right)^{\wedge} & \\
& \rightarrow H_{0}\left(P_{a, c}\left(\mathbb{P}^{1}-\{0,1, \infty\}\right), \mathbb{Q}\right)^{\wedge}
\end{aligned}
$$

are easily seen to be morphisms of MHS because of the formula (4).

By taking limit MHS, we can replace $a$ and $b$ by non-zero tangent vectors at the cusps. In particular, we have a mixed Hodge structure on

$$
H_{0}\left(P_{01, \overrightarrow{10}}\left(\mathbb{P}^{1}-\{0,1, \infty\}\right), \mathbb{Q}\right)^{\wedge}
$$

where $\overrightarrow{10}$ is the tangent vector $\partial / \partial(1-t)$ at $t=1$.

The following result is essentially a restatement of a result of Le and Murakami [26, Thm. A.9]. (See also, [15, Lemma 5.4].)

Lemma 20. The periods of the path $[0,1] \in P_{01,10}\left(\mathbb{P}^{1}-\{0,1, \infty\}\right)$ under the mapping

$$
\Theta_{\overrightarrow{01}, 1 \overrightarrow{0}}: H_{0}\left(P_{\overrightarrow{01}, \overrightarrow{10}}\left(\mathbb{P}^{1}-\{0,1, \infty\}\right)\right) \rightarrow A
$$

are mixed zeta numbers. All elements of MZN occur.

Proof. Let $\Phi \in A$ be the image of $[0,1]$ under the regularized period mapping $\Theta_{\overrightarrow{01}, \overrightarrow{10}}: H_{0}\left(P_{\overrightarrow{01}, \overrightarrow{10}} \mathbb{P}^{1}-\{0,1, \infty\}\right) \rightarrow A$. I claim that this is Drinfeld's associator [12]. The result will then follow as Le and Murakami have shown that the coefficients of the Drinfeld associator are mixed zeta numbers and all mixed zeta numbers occur.

To see that $\Phi$ is the Drinfeld associator, we use the prescription given in Corollary 5 for computing the regularized limit periods of a flat section. Applying this directly, we see that the renormalized value of $T([0,1])$ is

$$
\lim _{t \rightarrow 0} t^{X_{0}} T([t, 1-t]) t^{X_{1}}
$$

as the residue at $t=0$ of the connection for which $T[t, 1-t]$ is a flat section is

left multiplication by $X_{0}+$ right multiplication by $X_{1}$

The limit is a well-known expression for Drinfeld's associator. (Cf. [12], [26].)

Consider the paths illustrated in figure 1

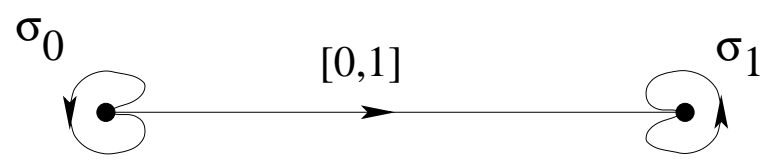

FiguRe 1.

where $\sigma_{0} \in P_{\overrightarrow{01}, \overrightarrow{01}} \mathbb{P}^{1}-\{0,1, \infty\}$ and $\sigma_{1} \in P_{\overrightarrow{10}, \overrightarrow{10}} \mathbb{P}^{1}-\{0,1, \infty\}$.

Theorem 19 now follows as one can check, as in the proof of the previous lemma, that under the renormalized homomorphism

$$
\Theta_{01}: \pi_{1}\left(\mathbb{P}^{1}-\{0,1, \infty\}, \overrightarrow{01}\right) \rightarrow A
$$

the image of the loop $\sigma_{0}$ is $\exp \left(2 \pi i X_{0}\right)$ and that the image of $\sigma_{1}$ under

$$
\Theta_{\overrightarrow{10}}: \pi_{1}\left(\mathbb{P}^{1}-\{0,1, \infty\}, \overrightarrow{10}\right) \rightarrow A
$$


is $\exp \left(-2 \pi i X_{1}\right)$. Since $\pi_{1}\left(\mathbb{P}^{1}-\{0,1, \infty\}, \overrightarrow{01}\right)$ is generated by $\sigma_{0}$ and $[0,1] \sigma_{1}[0,1]^{-1}$, and since

$$
T\left([0,1] \sigma_{1}[0,1]^{-1}\right)=\Phi e^{2 \pi i X_{1}} \Phi^{-1}
$$

we see that all periods of $\mathbb{Q} \pi_{1}\left(\mathbb{P}^{1}-\{0,1, \infty\}, \overrightarrow{01}\right)^{\wedge}$ lie in $\mathrm{MZN}_{\mathbb{C}}$.

\section{Mixed Tate Motives}

The question arises as to why one should expect the periods of $\pi_{1}\left(\mathbb{P}^{1}-\{0,1, \infty\}, \overrightarrow{01}\right)$ to be so special and to have such a nice description in terms of mixed zeta numbers. Deligne and Goncharov [11], building on previous work of both authors, especially [9] and several of Goncharov's papers in arXiv.org, have developed a satisfying and deep explanation in terms of the theory of mixed Tate motives.

Thanks to the work of Voevodsky [34], Levine [27], and Deligne-Goncharov [11], we now know that there is a tannakian category of mixed Tate motives over Spec of the ring of $S$-integers in a number field with the expected ext groups. So, in particular, there is a tannakian category of mixed Tate motives over Spec $\mathbb{Z}$. Such mixed Tate motives have a Hodge realization, which is a mixed Hodge structure, all of whose weight graded quotients are of type $(p, p)$. These mixed Hodge structures will have two $\mathbb{Q}$-structures — one coming from a $\mathbb{Q}$-de Rham structure, and another from topology (the "Betti realization"). The entries of the matrices relating them are, by definition, the periods of the motive.

Deligne and Goncharov $[\mathbf{1 1}]$ show that $\mathbb{Q} \pi_{1}\left(\mathbb{P}^{1}-\{0,1, \infty\}, \overrightarrow{01}\right)^{\wedge}$, equivalently the Lie algebra of the unipotent fundamental group of $\left(\mathbb{P}^{1}-\{0,1, \infty\}, \overrightarrow{01}\right)$, is a proobject in the category of mixed Tate motives over Spec $\mathbb{Z}$. One reason one should suspect this is that

$$
\mathbb{P}_{\mathbb{Z}}^{1}-\{0,1, \infty\}:=\operatorname{Spec} \mathbb{Z}\left[t, t^{-1},(1-t)^{-1}\right]
$$

has everywhere good reduction. However, the pointed variety $\left(\mathbb{P}_{\mathbb{Z}}^{1}-\{0,1, \infty\}, N\right)$, where $N \in \mathbb{Z}-\{0,1\}$, will not have good reduction at primes dividing $N$ as then the base point will move to a cusp. In order for $\left(\mathbb{P}^{1}-\{0,1, \infty\}, t\right)$ to have everywhere good reduction, we are forced to take $t$ to be $\overrightarrow{01}$, or one of its 5 other images under $\operatorname{Aut}\left(\mathbb{P}^{1},\{0,1, \infty\}\right)$.

The fact that $\mathbb{Q} \pi_{1}\left(\mathbb{P}^{1}-\{0,1, \infty\}, \overrightarrow{01}\right)^{\wedge}$ is a mixed Tate motive over Spec $\mathbb{Z}$ has a remarkable consequence for the transcendence properties of mixed zeta numbers $[\mathbf{3 3}]$ and for the Galois action on its $\mathbb{Q}_{\ell}$-form $[\mathbf{2 2}]$. In the first case, Zagier conjectured (unpublished), and Terasoma [33] has proved, that the dimension of the $\mathbb{Q}$-linear span of the mixed zeta numbers $\mathrm{MZN}_{m}$ of weight ${ }^{6} m$ is bounded by the dimension of the $m$ th weight graded quotient of the graded algebra

$$
\mathbb{Q}\left[Z_{2}\right] \otimes \mathbb{Q}\left\langle Z_{3}, Z_{5}, Z_{7}, \ldots\right\rangle
$$

where $Z_{m}$ has weight $m$ and $\mathbb{Q}\left\langle Z_{3}, Z_{5}, Z_{7}, \ldots\right\rangle$ denotes the free associative algebra generated by the $Z_{\text {odd }}$.

One currently unresolved question is whether the periods of all mixed Tate motives over Spec $\mathbb{Z}$ lie in $\mathrm{MZN}_{\mathbb{C}}$. Goncharov [14, p. 385] has conjectured that this is the case. To see why this might be, we need to discuss the motivic Lie algebra of Spec $\mathbb{Z}$ and its connection with the absolute Galois group $G_{\mathbb{Q}}:=\mathrm{Gal} \overline{\mathbb{Q}} / \mathbb{Q}$ and its action on $\mathbb{Q}_{\ell} \pi_{1}\left(\mathbb{P}^{1}-\{0,1, \infty\}, \overrightarrow{01}\right)^{\wedge}$.

\footnotetext{
${ }^{6}$ The weight of $\zeta\left(n_{1}, \ldots, n_{r}\right)$ is, by definition, $n_{1}+\cdots+n_{r}$.
} 
Since the category of mixed Tate motives over Spec $\mathbb{Z}$ is tannakian, it is the category of representations of a pro-algebraic group $\mathcal{A}$ over $\mathbb{Q}$. Since the tannakian fundamental group of the category of pure Tate motives over Spec $\mathbb{Z}$ is $\mathbb{G}_{m}$, this group is an extension

$$
1 \rightarrow \mathcal{U} \rightarrow \mathcal{A} \rightarrow \mathbb{G}_{m} \rightarrow 1
$$

where $\mathcal{U}$ is a prounipotent group. That the ext groups in the category of mixed Tate motives over $\mathbb{Z}$ have the desired relationship to $K_{\bullet}(\mathbb{Z})$ implies that the Lie algebra of $\mathcal{U}$ is a free pronilpotent Lie algebra on generators $z_{3}, z_{5}, z_{7}, \ldots$, where $z_{n}$ has weight $-2 n$, cf. $[\mathbf{1 1}]$ and [23]. The Lie algebra $\mathfrak{a}$ of $\mathcal{A}$ is itself a pro-object of the category of mixed Tate motives, and formal arguments show that all periods of mixed Tate motives over Spec $\mathbb{Z}$ occur as periods of $\mathfrak{a}$. Thus one should try to prove that all periods of the MHS of $\mathfrak{a}$ lie in $\mathrm{MZN}_{\mathbb{C}}$. One way to approach this is by studying the action of the absolute Galois group on the algebraic fundamental group of the thrice punctured line over $\overline{\mathbb{Q}}$.

Every mixed Tate motive $V$ has an $\ell$-adic realization for each rational prime $\ell$. This is a representation $\rho_{\ell}: G_{\mathbb{Q}} \rightarrow$ Aut $V_{\mathbb{Q}_{\ell}}$, where $V_{\mathbb{Q}_{\ell}}$ denotes $V \otimes_{\mathbb{Q}} \mathbb{Q}_{\ell}$. These actions induce a Zariski dense homomorphism

$$
G_{\mathbb{Q}} \rightarrow \mathcal{A}\left(\mathbb{Q}_{\ell}\right)
$$

through which $\mathcal{A} \otimes \mathbb{Q}_{\ell} \rightarrow$ Aut $V$ factors. The homomorphism $\rho_{\ell}$ is the composite of this with the canonical homomorphism $\mathcal{A} \rightarrow$ Aut $V$ :

$$
G_{\mathbb{Q}} \rightarrow \mathcal{A}\left(\mathbb{Q}_{\ell}\right) \rightarrow \text { Aut } V_{\mathbb{Q}_{\ell}}
$$

The image of $\mathcal{A} \otimes \mathbb{Q}_{\ell}$ in Aut $V_{\mathbb{Q}_{\ell}}$ is thus the Zariski closure of the image of $G_{\mathbb{Q}}$ (cf. $[23])$.

In the case of the unipotent fundamental group of $\left(\mathbb{P}^{1}-\{0,1, \infty\}, \overrightarrow{01}\right), \rho_{\ell}$ is the natural Galois action

$$
G_{\mathbb{Q}} \rightarrow \mathcal{A}\left(\mathbb{Q}_{\ell}\right) \rightarrow \operatorname{Aut} \mathbb{Q}_{\ell} \pi_{1}\left(\mathbb{P}^{1}-\{0,1, \infty\}, \overrightarrow{01}\right)^{\wedge}
$$

induced by the action of $G_{\mathbb{Q}}$ on its algebraic fundamental group. The Zariski closure of the image of this action is the image of the homomorphism

$$
\mathcal{A} \otimes \mathbb{Q}_{\ell} \rightarrow \operatorname{Aut} \mathbb{Q}_{\ell} \pi_{1}\left(\mathbb{P}^{1}-\{0,1, \infty\}, \overrightarrow{01}\right)^{\widehat{ }}
$$

This homomorphism is injective if and only if its derivative

$$
\mathfrak{a} \rightarrow \operatorname{Der} \mathbb{Q}_{\ell} \pi_{1}\left(\mathbb{P}^{1}-\{0,1, \infty\}, \overrightarrow{01}\right)^{\wedge}
$$

is. Since the periods of $\operatorname{Der} \mathbb{Q}_{\ell} \pi_{1}\left(\mathbb{P}^{1}-\{0,1, \infty\}, \overrightarrow{01}\right)^{\uparrow}$ lie in $\mathrm{MZN}_{\mathbb{C}}$, the periods of $\mathfrak{a}$ will too if the derivative is injective. At present it is not known whether or not this is the case, although there are computer results that show that in "small weights" the derivative is injective.

\section{References}

[1] J. Carlson: Extensions of mixed Hodge structures, in Journées de Géometrie Algébrique d'Angers, Juillet 1979, Sijthoff \& Noordhoff, Alphen aan den Rijn, 1980, 107-127.

[2] E. Cattani: Personal communication, April 17,2003.

[3] E. Cattani, A. Kaplan, W. Schmid: Degeneration of Hodge structures, Ann. of Math. (2) 123 (1986), 457-535.

[4] E. Cattani, A. Kaplan, W. Schmid: $L^{2}$ and intersection cohomologies for a polarizable variation of Hodge structure, Invent. Math. 87 (1987), 217-252.

[5] C. H. Clemens: Degeneration of Kähler manifolds, Duke Math. J. 44 (1977), 215-290. 
[6] P. Deligne: Équations différentielles à points singuliers réguliers, Lecture Notes in Mathematics, Vol. 163, Springer-Verlag, Berlin-New York, 1970.

[7] P. Deligne: Théorie de Hodge. II, Inst. Hautes Études Sci. Publ. Math. No. 40 (1971), 5-57.

[8] P. Deligne: Théorie de Hodge. III, Inst. Hautes Études Sci. Publ. Math. No. 44 (1974), 5-77.

[9] Le groupe fondamental de la droite projective moins trois points, Galois groups over $\mathbb{Q}$ (Berkeley, CA, 1987), 79-297, Math. Sci. Res. Inst. Publ., 16, Springer, New York, 1989.

[10] P. Deligne: Local behavior of Hodge structures at infinity, Mirror symmetry, II, 683-699, AMS/IP Stud. Adv. Math., 1, Amer. Math. Soc., Providence, RI, 1997.

[11] P. Deligne, A. Goncharov: Groupes fondamentaux motiviques de Tate mixte, preprint 2003, math.NT/0302267.

[12] V. Drinfeld: On quasitriangular quasi-Hopf algebras and on a group that is closely connected with $\mathrm{Gal}(\overline{\mathbb{Q}} / \mathbb{Q})$, (Russian) Algebra i Analiz 2 (1990), 149-181; translation in Leningrad Math. J. 2 (1991), 829-860

[13] J. Fay: Theta functions on Riemann surfaces, Lecture Notes in Mathematics, Vol. 352. Springer-Verlag, Berlin-New York, 1973.

[14] A. Goncharov: Polylogarithms in arithmetic and geometry, Proceedings of the International Congress of Mathematicians, Vol. 1, 2 (Zürich, 1994), 374-387, Birkhäuser, Basel, 1995.

[15] A. Goncharov: Multiple polylogarithms and mixed Tate motives, preprint math.AG/0103059.

[16] P. Griffiths: Periods of integrals on algebraic manifolds III, Some global differential-geometric properties of the period mapping, Inst. Hautes Études Sci. Publ. Math. No. 38 (1970), 125180.

[17] P. Griffiths, J. Harris: Principles of algebraic geometry, Wiley-Interscience, New York, 1978.

[18] R. Hain: The geometry of the mixed Hodge structure on the fundamental group, Algebraic geometry, Bowdoin, 1985 (Brunswick, Maine, 1985), 247-282, Proc. Sympos. Pure Math., 46, Part 2, Amer. Math. Soc., Providence, RI, 1987.

[19] R. Hain: Mixed Hodge structures on homotopy groups, Bull. Amer. Math. Soc. 14 (1986), 111-114.

[20] R. Hain: The de Rham homotopy theory of complex algebraic varieties II, K-Theory 1 (1987), 481-497.

[21] R. Hain: Iterated integrals and algebraic cycles: examples and prospects, Contemporary trends in algebraic geometry and algebraic topology (Tianjin, 2000), Nankai Tracts Math., 5, World Sci. Publishing, 2002, 55-118.

[22] R. Hain, M. Matsumoto: Weighted Completion of Galois Groups and Galois Actions on the Fundamental Group of $\mathbb{P}^{1}-\{0,1, \infty\}$, Compositio Math. to appear, math.AG/0006158.

[23] R. Hain, M. Matsumoto: Tannakian Fundamental Groups Associated to Galois Groups, preprint, math.AG/0010210.

[24] R. Kaenders: New Period Mappings for Plane Curve Singularities, preprint, math.AG/9802011.

[25] A. Kaplan, G. Pearlstein: Singularities of variations of mixed Hodge structure, preprint 2000, math.AG/0007040.

[26] T. Le, J. Murakami: Kontsevich's integral for the Kauffman polynomial, Nagoya Math. J. 142 (1996), 39-65.

[27] M. Levine: Mixed motives, Mathematical Surveys and Monographs 57, American Mathematical Society, 1998.

[28] E. Looijenga: $L^{2}$-cohomology of locally symmetric varieties, Compositio Math. 67 (1988), $3-20$.

[29] J. Morgan: The algebraic topology of smooth algebraic varieties, Inst. Hautes Études Sci. Publ. Math. No. 48 (1978), 137-204; Correction: Inst. Hautes Études Sci. Publ. Math. No. 64 (1986), 185.

[30] W. Schmid: Variation of Hodge structure: the singularities of the period mapping, Invent. Math. 22 (1973), 211-319.

[31] J. Steenbrink: Limits of Hodge structures, Invent. Math. 31 (1975/76), 229-257.

[32] J. Steenbrink, S. Zucker: Variation of mixed Hodge structure I, Invent. Math. 80 (1985), 489-542.

[33] T. Terasoma: Mixed Tate motives and multiple zeta values, Invent. Math. 149 (2002), 339369.

[34] V. Voevodsky, A. Suslin, E. Friedlander: Cycles, transfers, and motivic homology theories, Annals of Mathematics Studies 143, Princeton University Press, 2000. 
[35] W. Wasow: Asymptotic expansions for ordinary differential equations, Reprint of the 1976 edition. Dover Publications, Inc., New York, 1987.

[36] D. Zagier: Values of zeta functions and their applications, First European Congress of Mathematics, Vol. II (Paris, 1992), 497-512, Progr. Math., 120, Birkha" user, Basel, 1994.

[37] S. Zucker: Hodge theory with degenerating coefficients, $L_{2}$ cohomology in the Poincaré metric, Ann. of Math. (2) 109 (1979), 415-476. 